\title{
COMPARISON OF MUSCULOSKELETAL STRESS ON LUMBAR, SHOULDER, AND WRIST JOINTS WHILE MAKING A BED USING EASYTUCK PAD VERSUS OTHER BED-MAKING TOOLS
}

\author{
ARMIA ABDO' ${ }^{1}$, HANI AL-NAKHLI ${ }^{2 *}$ \\ ${ }^{1}$ AZA Health and Wellness Inc., Anaheim, California, US. ${ }^{2}$ Madinah General Hospital Complex, Saudi Arabia. Email: dr.alnakhli@gmail.com
} Received: 11 February 2020, Revised and Accepted: 14 February 2020

\section{ABSTRACT}

Objective: Currently, there are multiple bed-making tools utilized by consumers and businesses with the intention of increasing safety and reducing time to make a bed. In this study, we will compare musculoskeletal stress, time, and preference of making a bed using the EasyTuck pad versus three other bed-making tools and making a bed without any bed-making tool.

Methods: A sample of ten subjects performed the task of making a queen size mattress bed with one bottom fitted sheet and one top flat sheet, using the EasyTuck pad versus three other bed-making tools, and making a bed without any bed-making tool, in a random order. Time to make the bed, forces on the low back, shoulders and wrist, and the number of times each individual bent forward were measured during each trial. In addition, subjective preferences were measured using a simple questionnaire.

Results: Repeated measures analysis of variance was used to analyze the differences between the five different procedures. There were significant differences for all the measured variables. Regarding the time needed to make one bed, the EasyTuck pad had the least time range (80.6 s \pm 4.3 ), which was approximately $40 \mathrm{~s}$ less than making the bed with no tool $(\mathrm{p}<0.001)$. Furthermore, for the overall lumbar spine impulse force, the EasyTuck pad had the largest overall reduction in this category (90.6\%). Regarding the subjective data, the EasyTuck pad had an overall rating of 9.4 out of 10 , compared to the other tools with ratings of $5.9,5.1$, and 3.4 out of 10 , which was a statistically significant difference ( $\mathrm{p}<0.001$ ).

Conclusion: Making beds on a regular basis poses risk of injury, especially for those who are required to perform this for their occupation. However, using assistive tools for bed-making, could minimize, and prevent injuries and overload to the lower back, and other parts of the body, making the bed-making task safer. Based on our findings, the EasyTuck pad was by far the most efficient and safest tool to assist in making beds, with the lowest possible risk of injury when compared to all the other tools tested.

Keywords: Core muscles, Low back, Low back injury, Low back pain, Shoulder, Shoulder injury, Shoulder pain, Wrist, Wrist injury, Wrist pain, Muscle force, Force gauge, Stress, Threshold, EasyTuck Mat, BedMadeEZ, Bed tucker, ErgoTuck.

\section{INTRODUCTION}

Safety when making a bed is a major concern as there is currently no safe way to make abed, which poses a significant risk of injury and can create lifelong disabilities for certain professions such as hotel housekeepers, hospital staff, nursing home and in-home caregivers, and even for individual consumers.

"Seminal studies demonstrate that work-related bodily pain and injuries are significant problems. Very high proportions (77-91\%) of housekeeper's self-report pain primarily in their lower backs" [1]. "In 2010 , housekeepers had the highest rates of workers for overall injuries (7.9/100) and musculoskeletal disorders (3.2/100)" [2,3]. "Analyses of Workers Compensation data from a subset of unionized hotels revealed housekeepers' annual claims cost upward of $\$ 4.7$ million" $[4,5]$.

Currently, there are multiple bed-making tools utilized by consumers, and businesses with the intention of increasing safety, and reducing time to make a bed. In this study, we compared musculoskeletal stress on the low back, shoulders, and wrists while using four different bedmaking tools as well as making a bed without an assistive bed-making tool. We also compared the time, and steps needed to accomplish the bed-making task using each method. Subjects then filled out a questionnaire on their preference of methods.

\section{METHODOLOGY}

Design

Quantitative study

Participants

The study consisted of ten subjects.

\section{Inclusion criteria}

Healthy individuals between the ages of 20 and 60 years old were randomly recruited into this study. Demographics of the participants is shown in Table 1.

\section{Exclusion criteria}

Participants who had current symptoms in their upper extremities, and lower backs or had experienced back pain during the previous 3 months or had a history of the upper extremity or spinal surgery were excluded from the study. Furthermore, any subject on pain killers, or who were currently using any type of NSAID's was excluded from the study.

\section{Outcome measures}

The measurements of interest related to this study were: Low back, shoulder, and wrist extensors impulse forces to lift a queen size mattress to tuck in a fitted bed sheet and a flatbed sheet. The number of times needed to bend the low back, shoulders, and wrists to make the bed. The number of lifts needed to make the bed and the length of time for each lift. The steps needed to utilize each tool, the time to make a bed with each tool, and the subjective preference of the tools were also measured. These outcomes were collected while making a queen size bed with four bed-making tools as well as without any bedmaking tool.

\section{Impulse force}

A force gauge was used to determine the pressure needed to lift the mattress, to tuck in the sheets while making the bed. Each participants height (in cm's) and weight (in lbs) were collected. This data were used to determine the impulse forces with each lift using formula's A, B, and C, as listed in Fig. 1. [6] This formula is used for 
the low back, shoulder used to lift or use tool, and wrist used to lift or use tool.

\section{Procedure}

Each participant was asked to place the fitted sheet and the flat sheet on the bed, according to how they would do it at home when making a bed without a bed-making tool. They will then repeat the bed-making procedure with the four various tools utilizing the instructions that come with each product, no input will be provided outside of the directions that come with each tool. Each subject will make a bed 3 times with each bed-making tool, and 3 times without a bed-making tool. This will ensure that each participant increases competency of using each tool and will minimize user error that may impact data. The trial with the best time will be used to gather the data needed for time, and stress. Once the subjects start making the bed, they will not be given any direction or input to prevent influence.

The subjects will make each bed 3 times in a row and will randomly choose the order of which tool they will use by drawing numbers out of a hat from 1 to 5 . Each number coincides with a bed-making tool, they will continue to draw a number until all numbers have been used (Fig. 2).

Once they have completed making the bed using each tool, they will complete a questionnaire. They will simply be asked to fill it out without any direction to minimize bias or influence.

\section{Data analysis}

Statistical analysis was involved means, and Standard Errors of the Mean (SEM) for each variable. Changes between the five different measures were examined using repeated measures analysis of variance (ANOVA), and Bonferroni t-tests were used to assess pairwise comparisons. Data were analyzed using SPSS (version 22.0; IBM Corp, Armonk, NY), and Excel (Excel 2016) (Microsoft, Redmond, WA), and the level of significance was set at $\mathrm{p} \leq 0.05$.

Table 1: General demographics of the subjects

\begin{tabular}{lllll}
\hline & Age (years) & Height (cm) & Weight (lbs) & Gender \\
\hline Mean & 38.8 & 169.9 & 149.1 & 4 (male) \\
SEM & \pm 3.2 & \pm 3.5 & \pm 11.9 & 6 (female) \\
\hline
\end{tabular}

Significant differences between the five different procedures (making the queen size bed with and without the four bed-making tools) were observed. SEM: Standard error of the mean

\section{Equation A:}

Moment from the weight of the load $=(x$ lbs. $) x(y$ in. $)=z$ in-lbs Moment from the weight of the upper body $=(\mathrm{xlbs}$. $) \mathrm{x}(\mathrm{y}$ in. $)=\mathrm{z}$ in-lbs

Total Moment (clockwise) $=\mathrm{x}$ in-lbs

\section{Equation B:}

$\mathrm{x}$ in-lbs $=$ (Force generated by (erector spinae muscles, rotator cuff, wrist extensors) $)$ x ( 2 in.)

$(\mathrm{x}$ in-lbs $) /(2$ in. $)=($ Force generated by (erector spinae muscles, rotator cuff, wrist extensors))

$\mathrm{x}$ in-lbs = Force generated by (erector spinae muscles, rotator cuff, wrist extensors)

Equation C:

Force $\mathrm{X} \Delta$ Time=Impulse

Fig. 1: Impulse force equations

1. No tool

2. EasyTuck pad

3. BedMadeEZ wedge

4. Bed Tucker spatula

5. ErgoTuck spatula

\section{RESULTS}

1. The time to make the bed (in seconds).

a. The test used was a repeated measure ANOVA with a GreenhouseGeisser correction. The results determined that the EasyTuck pad had the highest decrease in the time to make the bed, with a statistically significant difference between the times (in seconds) to make the bed $(\mathrm{p}=0.001)$.

i. Post hoc comparison tests using the Bonferroni correction revealed a significant decrease in the time to make the bed using the EasyTuck pad versus No Tool (80.6 \pm 4.3 vs. $120.4 \pm 10.9$, respectively), which was statistically significant $(\mathrm{p}=0.015)$. This is equivalent to $33 \%$ decrease in time (approximately $40 \mathrm{~s}$ ) to make one bed with a bottom fitted sheet and a top flat sheet, when using the EasyTuck pad.

ii. Post hoc comparison tests using the Bonferroni correction revealed a significant decrease in the time to make the bed using the EasyTuck pad versus the BedMadeEZ wedge (80.6 \pm 4.3 vs. $97.9 \pm 4.6$, respectively), which was statistically significant $(\mathrm{p}=0.01)$. This is equivalent to $17.7 \%$ decrease in time (approximately $19 \mathrm{~s}$ ).

2. The number of bends in the lower spine to make one bed.

a. Repeated measures ANOVA with a Greenhouse-Geisser correction revealed that there was a statistically significant difference between the four tools as to the number of times each participant needed to bend their lower spine to make one bed $(\mathrm{p}<0.001)$

i. Post hoc comparison tests using the Bonferroni correction showed a significant decrease in the number of times that the participants had to bend their lower spine to make the bed when using the EasyTuck pad versus no tool $(7.1 \pm 0.3$ vs. $18.7 \pm 1.6$, respectively), which was statistically significant $(\mathrm{p}=0.001)$.

ii. Post hoc comparison tests using the Bonferroni correction showed a significant decrease in the number of times that the participants had to bend their lower spine to make the bed when using the EasyTuck pad versus BedMadeEZ wedge (7.1 \pm 0.3 vs. $10.4 \pm 0.6$, respectively), which was statistically significant $(\mathrm{p}=0.008)$.

3. The average time for each bend in the lower spine (in seconds)

a. Repeated measures ANOVA with a Greenhouse-Geisser correction verified that there was a statistically significant difference between the times (in seconds) for each bend in the lower spine while making the bed $(\mathrm{p}=0.001)$.

i. Post hoc comparison tests using the Bonferroni correction revealed a significant decrease in the times for each lower spine bend while making one bed using the EasyTuck pad versus no tool ( $6.7 \pm 0.5$ vs. $13 \pm 1.2$, respectively), which was statistically significant $(\mathrm{p}=0.004)$.

ii. Post hoc comparison tests using the Bonferroni correction revealed a significant decrease in the times for each lower spine bend while making one bed using the EasyTuck pad versus BedMadeEZ wedge ( $6.7 \pm 0.5$ vs.9.3 \pm 0.6 , respectively), which was highly significant $(\mathrm{p}=0.001)$.

iii. Post hoc comparison tests using the Bonferroni correction revealed a significant decrease in the times for each lower spine bend while making one bed using the EasyTuck pad versus the Bed Tucker spatula ( $6.7 \pm 0.5$ vs. $8.5 \pm 0.3$, respectively), which was statistically significant $(\mathrm{p}=0.019)$.

4. The average pounds of pressure on the lumbar spine.

a. Repeated measures ANOVA with a Greenhouse-Geisser correction determined that there was a statistically significant difference between the pounds of pressure exerted on the lumbar spine during the making of the bed once $(\mathrm{p}<0.001)$.

i. Post hoc comparison tests using the Bonferroni correction showed a significant decrease in the pounds of pressure

Fig. 2: Bed making tool ID 
exerted on the lumbar spine while making the bed when using EasyTuck pad versus no tool (4721.8 \pm 377 vs. $29084.4 \pm 3165.4$, respectively), which was highly significant $(\mathrm{p}<0.001)$

ii. Post hoc comparison tests using the Bonferroni correction showed a significant decrease in the pounds of pressure exerted on the lumbar spine while making the bed when using EasyTuck pad versus BedMadeEZ wedge (4721.8 \pm 377 vs. $7399.2 \pm 537.6$, respectively), which was statistically significant $(\mathrm{p}=0.003)$.

iii. Post hoc comparison tests using the Bonferroni correction showed a significant decrease in the pounds of pressure exerted on the lumbar spine while making the bed when using EasyTuck pad versus the Bed Tucker spatula (4721.8 \pm 377 vs. $6674 \pm 533.5$, respectively), which was statistically significant ( $\mathrm{p}=0.015)$.

iv. Post hoc comparison tests using the Bonferroni correction showed a significant decrease in the pounds of pressure exerted on the lumbar spine while making the bed when using EasyTuck pad versus ErgoTuck spatula (4721.8 \pm 377 vs. $6300.1 \pm 601.2$, respectively), which was statistically significant $(\mathrm{p}=0.041)$.

5. The average pounds of pressure on the shoulder

a. Repeated measures ANOVA with a Greenhouse-Geisser correction determined that there was a statistically significant difference between the pounds of pressure exerted on the shoulder during the making of the bed once $(\mathrm{p}<0.001)$.

i. Post hoc comparison tests using the Bonferroni correction showed a significant decrease in the pounds of pressure exerted on the shoulder while making the bed when using the EasyTuck pad versus no tool $(2402 \pm 202.1$ vs. $24678.9 \pm 2815.4$, respectively), which was statistically significant $(\mathrm{p}<0.001)$.

ii. Post hoc comparison tests using the Bonferroni correction showed a significant decrease in the pounds of pressure exerted on the shoulder while making the bed when using the EasyTuck pad versus the BedMadeEZ wedge ( $2402 \pm 202.1$ vs. $6546.7 \pm 510.8$, respectively), which was statistically significant $(\mathrm{p}<0.001)$.

iii. Post hoc comparison tests using the Bonferroni correction showed a significant decrease in the pounds of pressure exerted on the shoulder while making the bed when using the EasyTuck pad versus the Bed Tucker spatula ( $2402 \pm 202.1$ vs. $5894.8 \pm 499.9$, respectively), which was statistically significant $(\mathrm{p}<0.001)$.

iv. Post hoc comparison tests using the Bonferroni correction showed a significant decrease in the pounds of pressure exerted on the shoulder while making the bed when using the EasyTuck pad versus the ErgoTuck spatula (2402 \pm 202.1 vs. $5576.5 \pm 568.5$, respectively), which was statistically significant $(\mathrm{p}<0.001)$.

6. The average pounds of pressure on the wrist

a. Repeated measures ANOVA with a Greenhouse-Geisser correction determined that there was a statistically significant difference between the pounds of pressure exerted on the wrist during the making of the bed once $(\mathrm{p}<0.001)$.

i. Post hoc comparison tests using the Bonferroni correction, showed a significant decrease in the pounds of pressure exerted on the wrist while making the bed when using the EasyTuck pad versus no tool $(2259.8 \pm 197.2$ vs. $23207.7 \pm 2693.6$, respectively), which was highly significant $(\mathrm{p}<0.001)$.

ii. Post hoc comparison tests using the Bonferroni correction showed a significant decrease in the pounds of pressure exerted on the wrist while making the bed when using the EasyTuck pad versus the BedMadeEZ wedge (2259.8 \pm 197.2 vs. $6291 \pm 503.1$, respectively), which was highly significant $(\mathrm{p}<0.001)$. iii. Post hoc comparison tests using the Bonferroni correction showed a significant decrease in the pounds of pressure exerted on the wrist while making the bed when using the EasyTuck pad versus the Bed Tucker spatula $(2259.8 \pm 197.2$ vs. $5668.1 \pm 490.5$, respectively), which was highly significant $(\mathrm{p}<0.001)$.

iv. Post hoc comparison tests using the Bonferroni correction showed a significant decrease in the pounds of pressure exerted on the wrist while making the bed when using the EasyTuck pad versus the ErgoTuck spatula $(2259.8 \pm 197.2$ vs. $5366 \pm 559$, respectively), which was highly significant $(\mathrm{p}<0.001)$.

7. The overall impulse force on the lumbar spine

a. Repeated measures ANOVA with a Greenhouse-Geisser correction revealed that there was a statistically significant difference between the impulse forces on the lumbar spine while making the bed once $(\mathrm{p}<0.001)$.

i. Post hoc comparison tests using the Bonferroni correction showed a significant decrease in the impulse forces on the lumbar spine while making the bed once when using the EasyTuck pad versus no tool $(37341.2 \pm 4498.3$ vs. $396614.2 \pm 60118.6$, respectively), which was statistically significant $(\mathrm{p}=0.002)$.

ii. Post hoc comparison tests using the Bonferroni correction showed a significant decrease in the impulse forces on the lumbar spine while making the bed once when using the EasyTuck pad versus the BedMadeEZ wedge (37341.2 \pm 4498.3 vs. $76980.7 \pm 7573.5$, respectively), which was statistically significant $(\mathrm{p}=0.001)$.

iii. Post hoc comparison tests using the Bonferroni correction showed a significant decrease in the impulse forces on the lumbar spine while making the bed once when using the EasyTuck pad versus the Bed Tucker spatula (37341.2 \pm 4498.3 vs. $63946 \pm 6325.6$, respectively), which was statistically significant $(\mathrm{p}=0.003)$.

iv. Post hoc comparison tests using the Bonferroni correction showed a significant decrease in the impulse forces on the lumbar spine while making the bed once when using the EasyTuck pad versus the ErgoTuck spatula (37341.2 \pm 4498.3 vs. $61947 \pm 5114.5$, respectively), which was statistically significant $(\mathrm{p}=0.003)$.

8. The overall impulse force on the shoulder

a. Repeated measures ANOVA with a Greenhouse-Geisser correction revealed that there was a statistically significant difference between the impulse forces on the shoulder while making the bed once $(\mathrm{p}<0.001)$.

i. Post hoc comparison tests using the Bonferroni correction showed a significant decrease in the impulse forces on the shoulder while making the bed once when using the EasyTuck pad versus no tool $(5006.6 \pm 395$ vs. $33544.4 \pm 3634.3$, respectively), which was highly significant $(\mathrm{p}<0.001)$.

ii. Post hoc comparison tests using the Bonferroni correction showed a significant decrease in the impulse forces on the shoulder while making the bed once when using the EasyTuck pad versus the BedMadeEZ wedge (5006.6 \pm 395 vs. $15924.1 \pm 1141.8$, respectively), which was highly significant $(\mathrm{p}<0.001)$.

iii. Post hoc comparison tests using the Bonferroni correction showed a significant decrease in the impulse forces on the shoulder while making the bed once when using the EasyTuck pad versus the Bed Tucker spatula (5006.6 \pm 395 vs. $13021.2 \pm 971.6$, respectively), which was highly significant $(\mathrm{p}<0.001)$.

iv. Post hoc comparison tests using the Bonferroni correction showed a significant decrease in the impulse forces on the shoulder while making the bed once when using the EasyTuck pad versus the ErgoTuck spatula (5006.6 \pm 395 vs. 
$12222.9 \pm 1108.9$, respectively), which was highly significant $(\mathrm{p}<0.001)$

9. The overall impulse force on the wrist

a. Repeated measures ANOVA with a Greenhouse-Geisser correction revealed that there was a statistically significant difference between the impulse forces on the wrist while making the bed once $(\mathrm{p}<0.001)$.

i. Post hoc comparison tests using the Bonferroni correction showed a significant decrease in the impulse forces on the wrist while making the bed once when using the EasyTuck pad versus no tool $(4707.8 \pm 382.3$ vs. $31513 \pm 3433.7$, respectively), which was statistically significant $(\mathrm{p}<0.001)$.

ii. Post hoc comparison tests using the Bonferroni correction showed a significant decrease in the impulse forces on the wrist while making the bed once when using the EasyTuck pad versus the BedMadeEZ wedge $(4707.8 \pm 382.3$ vs. $15298.1 \pm 1119.3$, respectively), which was statistically significant $(\mathrm{p}<0.001)$.

iii. Post hoc comparison tests using the Bonferroni correction showed a significant decrease in the impulse forces on the wrist while making the bed once when using the EasyTuck pad versus the Bed Tucker spatula $(4707.8 \pm 382.3$ vs $12515.9 \pm 951.2$, respectively), which was statistically significant $(\mathrm{p}<0.001)$.

iv. Post hoc comparison tests using the Bonferroni correction showed a significant decrease in the impulse forces on the wrist while making the bed once when using the EasyTuck pad versus the ErgoTuck spatula (4707.8 \pm 382.3 vs. $11757.3 \pm 1089.9$, respectively), which was statistically significant $(\mathrm{p}<0.001)$.

Regarding the subjective data, there were two main categories:

1. Average overall discomfort (out of 10)

a. Repeated measures ANOVA with sphericity assumed determined that there was a statistically significant difference between the overall discomfort ratings while making one bed $(\mathrm{p}<0.001)$.

i. Post hoc comparison tests using the Bonferroni correction revealed a significant decrease in the overall discomfort rating while making one bed using the EasyTuck pad versus no tool $(0.133 \pm 0.054$ vs. $3.73 \pm 0.33$, respectively), which was highly significant $(\mathrm{p}<0.001)$.

ii. Post hoc comparison tests using the Bonferroni correction revealed a significant decrease in the overall discomfort rating while making one bed using the EasyTuck pad versus the BedMadeEZ wedge $(0.133 \pm 0.054$ vs. $3.17 \pm 0.35$, respectively), which was highly significant $(\mathrm{p}<0.001)$.

iii. Post hoc comparison tests using the Bonferroni correction revealed a significant decrease in the overall discomfort rating while making one bed using the EasyTuck pad versus the Bed Tucker spatula $(0.133 \pm 0.054$ vs. $2.07 \pm 0.21$, respectively), which was highly significant $(\mathrm{p}<0.001)$.

iv. Post hoc comparison tests using the Bonferroni correction revealed a significant decrease in the overall discomfort rating while making one bed using the EasyTuck pad versus the ErgoTuck spatula $(0.133 \pm 0.054$ vs. $2.03 \pm 0.14$, respectively), which was highly significant $(\mathrm{p}<0.001)$.

2. How likely to recommend the product (out of 10)

a. Repeated measures ANOVA with sphericity assumed determined that there was a statistically significant difference between the recommendations of the four products while assisting in making the bed $(p<0.001)$. As there were not any recommendations for not using a tool, this comparison was only made between the four tools.

i. Post hoc comparison tests using the Bonferroni correction revealed a significantly higher recommendation rating for making the bed using the EasyTuck pad versus the BedMadeEZ wedge ( $9.4 \pm 0.221$ vs. $3.4 \pm 0.79$, respectively), which was highly significant $(\mathrm{p}<0.001)$. ii. Post hoc comparison tests using the Bonferroni correction revealed a significantly higher recommendation rating for making the bed using the EasyTuck pad versus the Bed Tucker spatula ( $9.4 \pm 0.221$ vs. $5.9 \pm 0.53$, respectively), which was statistically significant $(\mathrm{p}=0.002)$.

iii. Post hoc comparison tests using the Bonferroni correction revealed a significantly higher recommendation rating for making the bed using the EasyTuck pad versus the ErgoTuck spatula ( $9.4 \pm 0.221$ vs. $5.1 \pm 0.41$, respectively), which was highly significant $(\mathrm{p}<0.001)$.

\section{DISCUSSION}

In the current investigation, it was clear that the EasyTuck pad was significantly superior to the rest of the bed-making tools in all the measured categories. There was a 33\% overall reduction of time to make the bed with the EasyTuck pad when compared to no tool, which was approximately a 40 s reduction.

Furthermore, regarding the steps needed to accomplish the bed-making task, all the tools as well as not using a tool needed two steps, whereas the EasyTuck pad only needed one step to make the bed. Furthermore, there was huge reduction in the time spent bending the lumbar spine when using the EasyTuck pad in comparison with no tool $(48.5 \%)$. Moreover, the only tool that had a significant decrease in the average time spent bending the shoulder and wrist joints when compared to no tool was the EasyTuck pad.

Turning to the lumbar impulse force affecting the individuals, the EasyTuck pad had the largest overall reduction in this category $(90.6 \%)$.

Finally, the EasyTuck pad scored the highest reduction in this segment with an $85 \%$ overall reduction.

\section{Limitations and recommendations}

Additional studies can be done for specific occupations that repeat this process daily, for example, hotel staff, nursing home staff, and hospital staff. Furthermore, increasing the sample size would be presumptive. Despite that, we provided evidence that using different assistive tools for bed-making could minimize injuries.

\section{CONCLUSION}

Making beds on a regular basis poses the risk of injury, especially for those who are required to perform this for their occupation. However, using assistive tools for bed-making could minimize and prevent injuries and overload to the lower back, and other parts of the body, making the bed-making task safer. Based on our findings, the EasyTuck pad is significantly more efficient, and it is also the safest tool for assisting in making beds, with the lowest possible risk of injury when compared to all the other tools.

\section{ACKNOWLEDGMENTS}

Funding for this study was provided by Instant Tuck USA, LLC. This company has had no input as to the format, methodology, and results of this study, and it has been completely independent from it. Special thanks for the participants in this study, as their time and efforts were critical for the successful completion of this study.

\section{REFERENCES}

1. Krause N, Scherzer T, Rugulies R. Physical workload, work intensification, and prevalence of pain in low wage workers: Results from a participatory research project with hotel room cleaners in Las Vegas. Am J Ind Med 2005;48:326-37.

2. Buchanan R, Kaufman A, Kung-Tran L, Miller EA. Genetic analysis of yeast $\operatorname{Sec} 24 \mathrm{p}$ mutants suggests cargo binding is not co-operative during ER export. Traffic 2010;11:1034-43.

3. Streit JM. Understanding and Improving Hotel Housekeeper Safety and Health: A Series of NIOSH Research Studies. In: Newsletter of the Society for Occupational Health Psychology. 2015. p. 7-8. 
4. Utterback DF, Schnorr TM. Use of Workers' Compensation Data for Occupational Safety and Health. United States: Department of Health and Human Services, Centers for Disease Control and Prevention, National Institute for Occupational Safety and Health; 2013.

5. Meyers A, Wurzelbacher S, Bertke S, Lampl M, Robins D, Bell J.
Using Workers Compensation Data for Surveillance of Occupational Injuries and Illnesses, Ohio, 2005-2009. Atlanta, Georgia: Centers for Disease Control and Prevention, NIOSH; 2009.

6. Cornell University Ergonomics; 2019. Available from: http://www. ergo.human.cornell.edu/dea3250flipbook/dea3250notes/lifting.html. 